\title{
Negative Reward Expectations in Borderline Personality Disorder Patients: Neurophysiological Evidence
}

Daniel Vega ${ }^{\mathrm{a}, \mathrm{b}, \mathrm{e}}$, Àngel Soto ${ }^{\mathrm{a}}$, Julià L Amengual ${ }^{\mathrm{c}}$, Joan Ribas ${ }^{\mathrm{a}}$, Rafael Torrubia ${ }^{\mathrm{b}}$, Antoni Rodríguez-Fornells, d,e,f, Josep Marco-Pallarés ${ }^{\mathrm{d}, \mathrm{e}, \mathrm{CA}}$

a Servei de Psiquiatria i Salut Mental, Hospital d'Igualada (Consorci Sanitari de l'Anoia) Igualada, Barcelona 08700, Spain.

${ }^{\mathrm{b}}$ Unitat de Psicologia Mèdica, Departament de Psiquiatria i Medicina Legal \& Institut de Neurociències, Universitat Autònoma de Barcelona, 08193 Bellaterra, Barcelona, Spain

${ }^{c}$ Neurodynamic Laboratory, Departament of Psychiatry and Clinical Psychobiology. Universitat de Barcelona, 08035, Barcelona, Spain

${ }^{\mathrm{d}}$ Dept. of Basic Psychology, Campus Bellvitge, University of Barcelona, L'Hospitalet de Llobregat, Barcelona 08097, Spain

${ }^{\mathrm{e}}$ Cognition and Brain Plasticity Group [Bellvitge Biomedical Research Institute- IDIBELL],

L'Hospitalet de Llobregat, Barcelona, 08097, Spain

${ }^{\mathrm{f}}$ Catalan Institution for Research and Advanced Studies, ICREA, Barcelona, Spain

\section{Corresponding author:}

Josep Marco Pallarés

Department Basic Psychology

Campus Bellvitge, University of Barcelona

FeixaLlarga s/n,

08907 L'Hospitalet (Barcelona), Spain

Phone number: +34 934034768

Fax: +34 934024268

E-mail: josepmarco@gmail.com 


\begin{abstract}
Borderline Personality Disorder (BPD) patients present profound disturbances in affect regulation and impulse control which could reflect a dysfunction in reward-related processes. The current study investigated these processes in a sample of eighteen BPD patients and eighteen matched healthy controls, using an event-related brain potentials methodology. Results revealed a reduction in the amplitude of the Feedback-Related Negativity of BPD patients, which is a neurophysiological index of the impact of negative feedback in reward-related tasks. This reduction, in the effect of negative feedback in BPD patients, was accompanied by a different behavioral pattern of risk choice compared to healthy participants. These findings confirm a dysfunctional reward system in BDP patients, which might compromise their capacity to build positive expectations of future rewards and decision making.
\end{abstract}




\section{Introduction}

Borderline Personality Disorder (BPD) is a complex and serious mental disorder with a characteristic pervasive pattern of instability on affect regulation, impulse control, interpersonal relationships and self-image, and severe functional impairment (Lieb, Zanarini, Schmahl, Linehan, \& Bohus, 2004). Although it seems to be a heterogeneous and less stable diagnosis (Zanarini, Frankenburg, Reich, \& Fitzmaurice, 2010), emotion dysregulation is the most permanent and frequent criterion (Carpenter \& Trull, 2013; Glenn \& Klonsky, 2009). Some influential accounts on the etiology of BPD propose that patients present an impairment in the processing of critical information in the adaptation of behavior to environmental contingencies (e.g., rewards and punishments associated with their actions) which would compromise their emotional self-regulation (Crowell, Beauchaine, \& Linehan, 2009). Nevertheless, studies on the processing of rewarding outcomes as well the expectations of receiving a reward have been scarce in these patients.

Emotional reactivity and cognitive control have been proposed as two features of the BPD emotional difficulties and, additionally, have been related to their attachment style which plays a central role in the development of the disorder (Agrawal, Gunderson, Holmes, \& Lyons-Ruth, 2004; Minzenberg, Poole, \& Vinogradov, 2008; Steele \& Siever, 2010). Rodent models and human neuroimaging have related the attachment system with the reward network due to a shared neural circuit which links a neuropeptide-sensitive mechanism (oxitocin/vasopressin), within the anterior hypothalamus, to the ventral tegmental area (VTA) and nucleus accumbens (see for a review Insel \& Young, 2001). In addition, from a gene-environment perspective, the dopamine DRD4 polymorphism in children has been related to disorganized attachment patterns with parents (Lakatos et al., 2000). The reward system is related to a variety of 
motivated behaviors and cognitive processes, such as reinforcement learning, novelty processing, action monitoring, decision making or addiction (Camara, RodriguezFornells, Ye, \& Münte, 2009). Therefore, the interaction between these two systems (reward and attachment) may be especially important for mediating the rewarding properties of social interaction as salient-motivating cue, and for affect and stress regulation (Strathearn \& Mayes, 2010; Vrticka, Andersson, Grandjean, Sander, \& Vuilleumier, 2008).

The idea of a dysfunctional reward system in the BPD has received growing theoretical interest in recent years (Bandelow, Schmahl, Falkai, \& Wedekind, 2010; Friedel, 2004). Previous research has reported impaired opioid activity, linked with the reward system (Prossin, Love, Koeppe, Zubieta, \& Silk, 2010). Furthermore, empirical data show that the BPD individuals make impulsive choices that result in fast appetitive rewards (Dougherty, Bjork, Huckabee, Moeller, \& Swann, 1999; Lawrence, Allen, \& Chanen, 2010). Several studies have suggested a dysfunctional reinforcement processing during both reward and loss feedbacks (Kirkpatrick et al., 2007; Völlm et al., 2007). A recent event-related brain potential (ERP) study (Schuermann, Kathmann, Stiglmayr, Renneberg, \& Endrass, 2011) showed reduced amplitude on the FeedbackRelated Negativity (FRN) component in BPD patients (relative to controls) who were performing an Iowa Gambling Task. Interestingly, this ERP component is elicited 250$300 \mathrm{~ms}$ after the presentation of a feedback, indicating a monetary loss or incorrect action (Gehring \& Willoughby, 2002; Miltner, Braun, \& Coles, 1997). The dynamics of the FRN have been explained using the reinforcement learning model (Holroyd \& Coles, 2002) which proposes that the FRN is indirectly reflecting the influence of decrease in VTA dopaminergic signals in the midbrain after unexpected punishments (Schultz, 1998). This reinforcing signal might be transmitted to the ventral striatum, as 
well as other cortical regions such as the medial prefrontal cortex. The FRN has been associated with a possible teaching signal concerning worse than expected consequences of actions. Considering this proposal, unexpected negative outcomes should elicit larger amplitude in the FRN component than unexpected positive outcome. In addition, several studies have described an enhancement of theta power activity after negative outcomes, which might not only be related to ACC activity, but also might reflect a broader neural network involved in orchestrating adaptive adjustments after errors or negative feedbacks (Cohen, Elger, \& Ranganath, 2007; Marco-Pallares et al., 2008). No previous research has studied theta power modulations in the BPD.

In the present study we evaluated the neurophysiological correlates (ERPs and theta oscillatory activity) associated with reward processing in a sample of BPD patients. In contrast to previous studies (Schuermann et al., 2011), we used a paradigm where the outcomes were not predictable, a monetary gambling task in which participants had to choose between two numbers in order to win or lose real money. In this paradigm the behavior is not guided by objective probabilities of receiving a reward or punishment (as for example, in reversal learning tasks or the Iowa Gambling Task; Schuermann et al., 2011), but by internal expectations as rewards and punishments are delivered at random. Therefore, we aimed to study the differences between BPD and healthy subjects associated with an uncertain environment or contexts in which clear predictions about the outcome of their actions were not possible. In addition, this paradigm has been shown to provide a very reliable FRN component and theta oscillatory activity in loss trials (Gehring \& Willoughby, 2002, Marco-Pallares et al. 2008, Marco-Pallares et al. 2009). We hypothesized that the characteristics of the present gambling task, in which there is neither correct response nor objective rule, could induce a differential behavioral pattern in BPD patients compared to healthy 
participants, especially in their risky choice patterns (that is, the tendency to increase their risk after certain outcomes; Gehring \& Willoughby, 2002; Padrao et al 2013). In addition, given the tendency of BPD to form unrealistic goals and negative expectations about the outcomes of their actions (Crowell, Beauchaine, \& Linehan, 2009), we hypothesized that monetary losses would have less impact in BPD patients than in healthy participants (reduced negative prediction error), yielding a reduction in the amplitude of the FRN component and theta oscillatory activity.

All these hypotheses were tested in a group of BPD women (double diagnostic interview by independent evaluators). Complementarily to the clinical instruments, and in order to better characterize the reward system in the sample and to control the individual differences in reward processing between patients and healthy participants, we used the Sensitivity to Reward and Punishment scales (Torrubia, Ávila, Moltó, \& Caseras, 2001), to measure approach-avoidance conflicts at cognitive level which could bias feedback processing (for a review on decision making and emotion regulation see Mitchell, 2011). Finally, as previous studies have shown that certain psychopharmacological drugs could affect the ERPs' components as well as the responsiveness of the reward brain system (see for example: Abler, Grön, Hartmann, Metzger, \& Walter, 2012; Johannes, Wieringa, Nager, Dengler, \& Münte, 2001) a protocol to assess total medication load, previously used in psychiatric samples (Vederman et al., 2012), was used to control possible confounding effects.

\section{Methods}

\section{Participants}

Thirty-six women ranging in age from 18 to 45 years old were included in the study. The BPD participants were 18 outpatients of the Psychiatry Department of the 
Hospital of Igualada (Barcelona, Spain) who met the diagnostic criteria according to DSM-IV-TR (APA, 2000). The Healthy Control (HC) group consisted of 18 healthy women recruited via local advertisement without history of any psychiatric disorder. The exclusion criteria were the presence of brain injury, psychotic, bipolar, or current major depressive disorder, drug or alcohol abuse in the previous month, and an Intelligence Quotient (IQ) below 80. Groups were matched by age and IQ. The participants were paid, and the study followed the Declaration of Helsinki and was approved by the local Scientific and Ethics Committee.

The BPD patients underwent a double diagnostic interview by independent evaluators trained in the administration of the Spanish validation of the Diagnostic Interview for Borderlines-Revised (Barrachina et al., 2004), in order to ensure the diagnosis. Both BPD and HC groups were assessed with a Spanish adaptation of the Structured Clinical Interview for DSM-IV Axis II Personality Disorders (Pérez-Prieto et al., 2008) and for DSM-IV Axis I (First \& Gibbon, 1997). The BPD depressive symptoms ranged from 4 to $17(M=11.55, S D=4.27)$ in the Hamilton Depression Rating Scale (HDRS, Hamilton, 1960). Medication prescription in the BPD group was stable along the study $(M=2.33, S D=1.84$, range: $0-5)$. The selective serotonin reuptake inhibitors $(N=10)$ and benzodiazepines $(N=9)$ were the most used, followed by mood stabilizers $(N=7)$, atypical antipsychotics $(N=4)$ and another type of drugs such noradrenergic and serotoninergic antidepressants $(N=5)$. Demographic and clinical variables can be observed in table 1 .

\section{Materials}

Self-report measures. The Sensitivity to Punishment and Sensitivity to Reward Questionnaire (SPSRQ, Torrubia et al., 2001) is a questionnaire developed and validated directly on Gray's personality model (Corr, 2004) and consists of two scales: 
the Sensitivity to Punishment scale (SP), which measures individual differences on Behavioral Inhibition System functioning, and the Sensitivity to Reward scale (SR), which measures individual differences on Behavioral Activation System functioning.

To assess the assigned value given by participants to a determined amount of money, a scale was created ad hoc. It consisted of four visual analog scales (VAS) which ranged from 0 to 100 points. The first two aimed to assess the subjective impact produced by the possibility of receiving a certain amount of money (100 euro and .50 euro cent), and the others were used for the assessment of the subjective impact produced by the possibility of losing a given amount of money (100 euro and .50 euro cent). High scores indicated that participants evaluated the impact of a possible loss/gain as very important for themselves. This measure aimed to capture the impact of possible economic feedbacks considering four possibilities (depending on valence and magnitude) in a daily virtual scenario.

Medication Load. This scale is a protocol to assess total medication load, previously used in psychiatric samples (Vederman et al., 2012). For the implementation, anti-depressant, anxiolytic, mood stabilizer, and anti-psychotic medications were coded as absent $=0$, low $=1$, or high $=2$, based on previously employed methods to convert each medication to a standardized dose (Almeida et al., 2009; Sakheim, 2001). Antipsychotics were converted into chlorpromazine dose equivalents (Davis \& Chen, 2004). As a result, we obtained a composite measure of total medication load by summing all individual medication codes for each individual medication within categories for each BPD patient.

Gambling Task. A monetary gambling task similar to the one described by Gehring \& Willoughby (2002) was used (see Figure 1A). In this task two numbers (25 and 5) were presented on a computer screen. Participants had to make an obligatory left 
or right mouse button press response with their right index-finger, indicating the number they wanted to bet. For instance in case of a [25][5] display, a left button press indicated the selection of the number 25 , and a right button press the selection of the number 5 . After the selection, one of the numbers turned red while the other turned green. If the number selected changed to red, the participant lost the corresponding amount in Euro cents, whereas if subject selected the green number he won this amount in Euro cents. After two seconds, the following trial began with the presentation of a warning signal (“*”; 500 ms duration), followed by a new set of numbers. Participants began the task with an initial 1,000 points $(1$ point $=1$ Euro cent $)$ and were encouraged to gain as much as possible and were familiarized with the task during a brief practice block.

The experiment comprised 17 blocks with 40 trials each, with the mean expected value of monetary outcome of zero on each block, to avoid potential confounding influences of a differential probability of gains or losses. Every 10 trials, the accumulated amount of money was presented for 7 seconds, and at the end of the experiment, the participants were paid the final amount.

\section{Procedure}

The clinical interviews (DIB-R only BPD group) and self-reported, intelligence and socio-demographical were gathered by a trained clinicians.

EEG (Synamps, Neuroscan) was recorded at $250 \mathrm{~Hz}$ sampling rate $(0.01 \mathrm{~Hz}$ high pass filter, $50 \mathrm{~Hz}$ notch filter) using tin electrodes mounted in an elastic cap and located at 29 standard positions (Fp1/2, Fz, F7/8, F3/4, Fc1/2 Fc5/6, Cz, C3/4, T7/8, $\mathrm{Cp} 1 / 2, \mathrm{Cp} 5 / 6, \mathrm{Pz}, \mathrm{P} 3 / 4, \mathrm{P} 7 / \mathrm{P} 8, \mathrm{Po} 1 / 2, \mathrm{O} 1 / 2)$ while participants were performing the gambling task. Biosignals were referenced off-line to the mean of the activity at the two mastoid processes. Vertical eye movements were monitored with an electrode at the 
infraorbital ridge of the right eye. Electrode impedances were kept below $5 \mathrm{k} \Omega$ during all the register.

\section{Statistical analysis}

Firstly, descriptive data analyses were carried out. Differences between groups concerning baseline demographic, diagnostic characteristics, and self-report data, were tested using Pearson's Chi-square test $\left(\chi^{2}\right)$ for the categorical variables and two-tailed independent Student's $t$-test to compare means. Bivariate correlations were used to measure the association between continuous variables.

Differences in risky pattern behavior between groups in the gambling task were analyzed using repeated-measures analyses of variance (ANOVA) with two withinsubjects factors (Feedback valence in the previous trial [gain, loss] and Feedback magnitude in the previous trial [large, small]) and one between subject factor (group, BPD vs. HC). Reaction times were analyzed using an ANOVA analysis with one within-subject factor (Bet magnitude [25/5]) and one between-subjects factor (group, BPD vs. HC).

EEG was lowpass filtered off-line to $40 \mathrm{~Hz}$ and feedback-locked ERPs were averaged from $100 \mathrm{~ms}$ prior to the feedback (baseline) to $1000 \mathrm{~ms}$ after it. Epochs exceeding $\pm 100 \mu \mathrm{V}$ in EOG or EEG were removed from further analysis. To study the time-frequency behavior of the electrical activity elicited by the feedback, four-second epochs were generated (2000 $\mathrm{ms}$ before and after the feedback stimulus). Epochs exceeding $\pm 100 \mu \mathrm{V}$ in EOG or EEG were removed from further analysis. Single-trial data was convoluted using a 7 cycles complex Morlet wavelet. Changes in the time varying energy (square of the convolution between wavelet and signal) in the studied frequencies (from $1 \mathrm{~Hz}$ to $40 \mathrm{~Hz}$; linear increase) with respect to baseline were computed for each trial and averaged for each subject before performing a grand average. For the 
FRN, repeated-measures ANOVA with Valence (gain, loss), Magnitude (large, small) and electrode location $(\mathrm{Fz}, \mathrm{Cz}, \mathrm{Pz})$ as within-subject factors and group (BPD, HC) as between-subject factor was performed introducing the mean amplitude at the 260-300 ms time-window after feedback presentation (Marco-Pallarés et al., 2008). For wavelet analysis, we used a time-frequency range based in the maximum differences between gains and losses (200-300 $\mathrm{ms}$ and $300-450 \mathrm{~ms}$ after feedback presentation). The Greenhouse-Geisser epsilon correction was used when appropriate.

\section{Results}

\section{Psychometric scales}

The results of the psychometric scales are shown in table 1. No significant differences were found on the VAS scales, indicating no between-group differences in the assigned value to a determined amount of money. Furthermore, the Sensitivity to Reward and the Sensitivity to Punishment were significantly higher in the BPD group than in the control group.

\section{Behavioral Results}

Participants tended to bet 25 more than 5, both in the control $(56.4 \pm 10.0 \%)$ and in the BPD $(56.0 \pm 9.4 \%)$ group. There were no significant differences among groups in percent of 25 choices $(t(34)=.1, p=0.5)$. However, when analyzing the pattern of risky choices considering previous outcome (based on Gehring \& Willoughby, 2002), a differential behavior pattern among groups was observed (Figure 1B). Repeated-measures ANOVA with two within factors (valence and magnitude) and one between-subjects factor (group) revealed a significant main effect for magnitude $(F(1,34)=4.4, p=0.04)$, which was significantly different in the two groups (magnitude $\mathrm{x}$ group, $F(1,34)=5.6, p=0.02)$. Therefore, as shown in Figure $1 \mathrm{~B}$, this 
interaction indicated that control participants increased their risk (betting more on 25 than 5) after winning or losing the largest amount of money (25; magnitude effect for control participants, $F(1,17)=6.5, p=0.02$ ). In contrast, BPD patients did not show this adjustment pattern, and bet independently from the outcome of the previous trial (magnitude effect, $F(1,17)=.08, p=0.8$, see Figure $1 \mathrm{~B}$ ).

In addition we also found a marginal significant valence $\mathrm{x}$ magnitude effect $(F(1,34)=3.5, p=0.07)$ but without a group effect (valence $\mathrm{x}$ magnitude $\mathrm{x}$ group $F(1,34)=0.564, p=0.5)$. Neither valence $(F(1,34)=1.3, p=0.3)$ nor the interaction between valence and group $(F(1,34)=1.5, p=0.2)$ yielded further significant effects. Thus, regarding trial-by-trial risk-sequential adjustments, the choices of the BPD group were uninfluenced by the outcome received in the previous trial, a pattern that is clearly different from the one observed in the control group and from the results obtained in previous investigations (Camara et al., 2010; Gehring \& Willoughby, 2002; Masaki, Takeuchi, Gehring, Takasawa, \& Yamazaki, 2006; Padrao et al., 2013).

Additionally, a reaction time analysis was conducted. The ANOVA revealed a marginal main effect of the bet magnitude $(F(1,34)=3.9, p=.06)$ indicating a fast betting to 25 than 5 . No significant bet magnitude $\mathrm{x}$ group interaction was found (BPD: bet $25, M=696 \mathrm{~ms}, S D=236$; bet $5, M=725 \mathrm{~ms}, S D=280$; HC: bet $25, M=652 \mathrm{~ms}$, $S D=324$; bet $5, M=687 \mathrm{~ms}, S D=338 ; F(1,34)=.04, p=0.8)$.

\section{ERP data}

Figure 2A shows the Event Related Potentials associated with the four different feedback conditions (gain 25, gain 5, loss 25, loss 5). In the 260-300 ms time range the negative feedbacks (monetary losses) presented a negative deflection compared to monetary gains compatible with the FRN ERP (Gehring \& Willoughby, 2002; MarcoPallares et al., 2008). A repeated measures ANOVA carried out at this time range with 
feedback valence (gain/loss), feedback magnitude (25/5) and electrode location (Fz, Cz, $\mathrm{Pz}$ ) as within-subject factors and Group (BPD/Control) as between-subject factor revealed a main significant effect of valence $(F(1,34)=40.1, p<0.001)$, indicating the increase of negativity observed after negative feedbacks. This effect presented a standard frontocentral topography (see Figure 2B) as revealed by a significant valence $\mathrm{x}$ electrode interaction $(F(2,68)=16.0, p<0.001)$. Analysis also revealed a significant magnitude effect $(F(1,34)=7.3, p=0.01)$, indicating an increase in activity for large as compared to small feedbacks $(25>5)$.

Figure $2 \mathrm{~B}$ shows the different waveforms (monetary loss minus monetary gains) for the $\mathrm{Fz}$ and $\mathrm{Pz}$ electrodes. The control group presented a larger FRN than the BPD (significant valence $\mathrm{x}$ group interaction, $F(1,34)=4.5, p=0.04)$. Post-hoc analyses revealed no significant differences between groups in the gain $(t(34)=1.0, p=0.3)$, nor in loss conditions $(t(34)=-0.07, p=0.9)$, but in the loss minus gain condition $(t(34)=-$ 2.13, $p=0.04)$. Interestingly, previous studies have suggested that the difference waveform is the best marker of the FRN processing (Holroyd 2004). Comparison between gain and loss trials was significant in both control $(t(17)=6.92, p<0.001)$ and BPD participants $(t(17)=2.64, p=0.02)$. Finally, in order to discard any effect associated to the medication, we analyzed the medication load, including it as a covariate in the repeated-measures ANOVA in the BPD group. There was no significant valence $\mathrm{x}$ load interaction $(F(1,16)=1.2, p=0.3)$.

\section{Time-frequency}

Figure 3 shows the power changes at frequencies between 1 to $40 \mathrm{~Hz}$ associated with positive and negative feedbacks for the control (Figure 3A) and the BPD (Figure 3B) group at the Fz electrode. Monetary losses were characterized by greater theta band activity $(3-7 \mathrm{~Hz})$ for negative feedback compared to positive feedback. We analyzed 
two different time ranges at this frequency band: $200-300 \mathrm{~ms}$ and $300-450 \mathrm{~ms}$ after feedback presentation. In the former time range, we found a significant valence $\mathrm{x}$ electrode interaction $(F(2,68)=19.7, p<0.001)$, showing an increase in theta band for loses compared to gains at frontocentral electrodes (Figure $3 \mathrm{~A}$ and $3 \mathrm{~B}$ ), but not a main valence effect $(F(1,34)=0.1, p=0.7)$. There was no significant effect of group in the valence (valence $\mathrm{x}$ group, $F(1,34)=2.4, p=0.13$; valence $\mathrm{x}$ electrode $\mathrm{x}$ group, $F(2,68)$ $=2.1, p=0.14)$. We also found a significant magnitude effect in this time range $(F(2,68)=8.2, p<0.01)$ but not a significant interaction between magnitude and electrode $(F(2,68)=1.9, p=0.2)$. None of these interactions yielded a significant group effect (magnitude $\mathrm{x}$ group, $F(1,34)=1.1, p=0.3$; magnitude $\mathrm{x}$ electrode $\mathrm{x}$ group, $F(2,68)=0.3, p=0.8)$. All the other effects were not significant $(F<1.6, p>0.2)$.

Then we analyzed the 300-450 ms time range. Again, frontocentral electrodes showed a greater theta power for losses than gains (valence $\mathrm{x}$ electrode, $F(2,33)=18.8, p<$ 0.001), and the corresponding ANOVA revealed significant differences between control and BPD groups in the 3-7 Hz and 300-450 ms time-frequency range (valence $\mathrm{x}$ group, $F(1,34)=4.8, p=0.04)$, indicating that the difference between gains and losses in the control group was higher than in the BPD group. Post-hoc analyses again revealed no significant differences between groups in the gain $(t(34)=-1.3, p=0.2)$, nor in loss conditions $(t(34)=-0.07, p=0.9)$, but in the loss minus gain condition $(t(34)=2.2, p=$ 0.04). However, neither controls $(t(17)=-1.36, p=0.2)$, nor BPD patients $(t(17)=1.72, p$ $=0.1)$ showed significant differences between gain and loss conditions. In addition, we found a marginal significant interaction of valence and medication load for valence in the BPD group (valence $\mathrm{x}$ load $F(1,16)=3.1, p=0.099$ ). In order to determine the origin of this marginal effect, we divided the medication load between different groups: antidepressants, anxiolytics, antipsychotics and anticonvulsants. We did not find 
significant differences with any of the specific medication types (antidepressants, $F(1,16)=0.9, p=0.4 ;$ antipsychotics, $F(1,16)=1.3, p=0.3 ;$ anxiolytics, $F(1,16)=1.1$, $p=0.3 ;$ anticonvulsants $F(1,16)=2.6, p=0.13)$.

\section{Discussion}

Reward-related feedback processing in a group of BPD patients was evaluated, analyzing behavioral adjustments (change in risky patterns), the feedback related negativity ERP component (FRN) and the time frequency decomposition of EEG after receiving monetary gains and losses (theta band power). A decrease in the amplitude of the FRN component and of the power of theta activity for the BPD group in comparison to the control group was encountered, suggesting an altered pattern of negative feedback processing which could indicate an impairment in the reward system of BPD patients. This deficit might not only be related to the valence, but also to unexpectedness of the outcome which might lead the patients to an incapacity for adjusting their behaviors and making predictions according to the history of previous outcomes.

These results are only partially in line with previous research findings (Kirkpatrick et al., 2007; Völlm et al., 2007) which have suggested an altered reward processing in the BPD patients, following both positive and negative feedback (compared with controls). Interestingly, a recent study by Schuermann et al. (2011) using an Iowa Gambling Task has shown that BPD patients made more risky choices than healthy participants and did not improve their performance nor learn during the task. Therefore BPD patients showed a reduced ability to learn from feedback. In addition, BPD patients also showed reduced FRN amplitude following both positive and negative feedbacks. Our results also suggest that BPD patients present an impairment in behavioral pattern indicated by the lack of adjustment after large magnitude gains and 
losses, but without an increase in the percentage of high magnitude bets. In addition, our study showed a reduction in the FRN amplitude (Schuermann et al. 2011) and theta oscillatory activity (the latter, however, correlating with medication load).

The FRN and theta activity reduction found in the BPD group could indicate a reduction in the prediction error after the negative feedback, which could be yielded by a reduced impact of the losses in BPD patients and/or a greater expectancy of receiving punishments (Hajcak, Moser, Holroyd, \& Simons, 2007). These results are of great importance because correct processing of the environment contingencies (rewards and punishments) is required for the formation of suitable predictions and expectations, which will optimize the behavioral adaptation. In this context, the FRN component indexes the motivational impact of the outcome event more than the information content of the negative feedback (Gehring \& Willoughby, 2002). More specifically, Holroyd and Coles (Holroyd \& Coles, 2002) proposed that both the FRN as well as the theta activity increase appears after worse than expected results of our actions, which might be related to a brain signature conveying information of a prediction error; that is, the discrepancy between the real and the expected outcome of our actions (Cavanagh, Cohen, \& Allen, 2009; Chase, Swainson, Durham, Benham, \& Cools, 2011; Talmi, Fuentemilla, Litvak, Duzel, \& Dolan, 2012). Therefore, when negative feedback is unexpected or the loss is greater than predicted, the FRN and theta activity would be higher, as is the difference between real and expected outcome. However, it is important to note that recently a new interpretation of the FRN has been proposed (Holroyd, Pakzad-Vaezi \& Krigolson, 2008). According to this account, negative feedbacks would produce a standard N200 (the FRN) and, in contrast, positive feedbacks would elicit a positive-going deflection which would superpose to the N200-FRN, reducing its amplitude. Therefore, the important effect would be the reduction of FRN with positive 
outcomes, constituting the so-called feedback correct-related positivity (fCRP). Following a similar rationale, Hajihosseini \& Holroyd (2013) proposed that the activity in the ACC after unexpected positive outcomes would reduce both the theta oscillatory activity and the N200 Event-Related Potential in gain trials. According to this interpretation, the reduction in the FRN found in BPD patients could be explained by a reduction in the N200 amplitude due to decreased novelty processing associated with both gain and loss events (see, e.g., Folstein \& Van Petten, 2008, for a N200 review). In other words, this account would suggest that BPD patients would be less sensitive to the novel impact associated to the feedback processing. However, there is still an open debate on the interpretation of the FRN-fCRP ERP components and more studies are needed in order to establish a correct functional interpretation for these responses.

The BPD group scored high both in SR and SP. Thus, while the high SR scores could indicate a pervasive tendency to pursue fast appetitive rewards, at the same time, the high scores on SP could suggest an underestimation of potential rewards and overestimation of possible risks, punishment or non-rewarding outcomes (Corr, 2002). This combination, in addition to alterations in the feedback processing (FRN), could lead them to constant conflicts at the cognitive level and emotional instability which was indirectly shown by the SPSRQ (Amodio, Master, Yee, \& Taylor, 2008). To complement the SCSRQ, we created ad hoc a VAS. These scales did not show between groups differences, supporting a similar importance given to the possibility of receiving/losing a particular amount of money. This result combined with the scores of SPSRQ suggests that the reduction of FRN and theta activity is not related to a reduction of the impact of losses (as BPD patients show increased SP values) but more likely linked to an increase in the expectancy to lose. 
The present results might reflect impairment in the mesolimbic dopaminergic system (Marco-Pallarés et al., 2009), in line with neuroimaging findings (see for a review Mauchnik \& Schmahl, 2010). In addition, some theoretical approaches to borderline etiology (Bandelow et al., 2010; Friedel, 2004) as well as some clinical traits such as emotion dysregulation or impulsivity, psychotic-like symptoms and partial efficacy of antipsychotic drugs among others, also suggest a deregulation of the reward system in these patients. Furthermore, the current results are in line with previous research showing that the Error Related Negativity, a parallel component which appears after the commission of an error (Falkenstein, Hohnsbein, Hoormann, \& Blanke, 1991; Gehring, Goss, Coles, Meyer, \& Donchin, 1993) is also reduced in BPD patients, suggesting an impaired capacity to learn from errors and to implement sequential cognitive control adjustments (de Bruijn et al., 2006; Ruchsow et al., 2006). It is important to note that, according to the reinforcement learning theory (Holroyd \& Coles, 2002), the FRN acts as a teaching signal after worse than expected events (negative prediction error, but see Holroyd et al., 2008) and it might be used to reinforce correct responses and inhibit erroneous ones. Impairment of this signal might result in non-optimal adaptation of behavior after errors or negative feedbacks. While in the present experiment there is no correct strategy per se (as rewards and punishments were delivered at random without participants' knowledge), differences in the behavioral adjustments (risk patterns) between control and BPD group supports this idea (see Figure 1B). The risk pattern in the control group is very similar to the one found in Padrao et al, 2013 (but see Gehring \& Willoughby, 2002). In the two studies, control participants showed an increase in their risky decisions characterized by a greater selection of high magnitude choices after large magnitude outcomes (whether monetary gains or losses). Interestingly, this patter differs from the one shown in Gehring \& 
Willoughby (2002), in which the risky-choice pattern increased linearly, from high gains to high losses. However, it is important to note that both experimental paradigms are slightly different, the current paradigm being a simplified version of the Gehring \& Willoughby (2002) (see Marco-Pallares et al, 2008). In contrast, BPD patients showed a flat risky-choice pattern, with similar percentage of high magnitude selection after any outcome. This behavior seems to suggest that patients did not use previous information and bet independently from the outcome of the previous trials. This result is also similar to the reduced risky choices after large magnitude trials in participants with high values in the anhedonia trait (Padrao et al., 2013). In addition, patients with high pathological anxiety also show a reduced tendency to risk, especially after small gains (Giorgetta, et al. 2012). Other studies have shown that schizophrenic patients reduce the exploration of uncertain scenarios with higher risk (Strauss et al., 2011). It has also been proposed that the decrease of risk-taking behavior might be related to reduced expectations of reward in the future (pessimistic evaluation of future, Giorgetta et al., 2012). The present results do not show a global reduction in risk-taking behavior (the percentage of choosing 25 is the same in the two groups), but in the pattern of risky choice after different outcomes. Therefore, the lack of a sequential adjustment strategy in these patients could be explained by a reduced impact of previous trial feedback and a subsequent impairment in the activation of automatic adjustment mechanisms elicited most probably in the medial prefrontal cortex (Cavanagh et al., 2009; Ridderinkhof, Ullsperger, Crone, \& Nieuwenhuis, 2004; Schuermann et al., 2011). However in the present paradigm the selection of the magnitude is not an independent measure, but it depends on the choice of the participant. This makes difficult to establish a causal link between the magnitude of the previous outcome and the current selection, as they are not completely independent. In addition, it is important to note that both groups ended 
with similar amounts of money, that is, both performed the task equally well. Therefore it cannot be stated which was the more correct strategy, although risky choice pattern in healthy controls was similar to previous studies (Padrao et al., 2013).

The alteration of reward processing and adjusting behavior found in present results might help understand the tendency of BPD patients to make suboptimal, even disadvantageous, decisions. Moreover, the results are in accordance with some theories about the development of BPD psychopathology, which propose that early environmental factors (i.e. invalidating developmental context or neglect) (Linehan, 1993), as well as genetic factors, could alter the reward pathways in the brain and cause "hyper-reactivity" of the attachment system (Fonagy \& Bateman, 2006). This phenomenon is a vicious circle between attachment style and environmental experiences, resulting in certain cognitive biases which complicate the decision making based on previous experiences and feedback, in line with current cognitive therapy proposals (Clark \& Beck, 2010). Interestingly, our experimental context could be considered as an experimental model of an invalidating setting similar to that proposed by Linehan in her biosocial theory of BPD (see for a review Crowell, Beauchaine, \& Linehan, 2009), in which an inconsistent use of punishment and reward by progenitors was postulated. In contrast to other experimental approaches in which risk conditions or specific rule probabilities were used (Schuermann et al., 2011), in the present study participants neither knew the probability of each choice nor whether a correct strategy existed or not. Therefore, the uncertainty created by the gambling task (winning or losing 5 or 25 at random while participants are trying to "maximize" their gains) might generate an ambiguous situation after the participants' choice, as they did not have any evidence or signal to trust in their election or strategy, which in patients might impair the capacity to use the history of previous outcomes to adjust the behavior. 
The main limitation of present study arises from the fact that the BPD patients were on medication during the study which could affect the effects in brain electrical activity. However, it is important to note that the prescription was stable along the assessment process, and that the symptoms of unmedicated BPD patients could hinder (even make impossible) the experiment performance. Despite this, we have included a standardized measure given that previous findings have suggested an effect of several psychopharmacological drugs on for example, action monitoring (Riba, RodriguezFornells, Munte, \& Barbanoj, 2005) or reward processing (Abler, Grön, Hartmann, Metzger, \& Walter, 2012). Thus, we found only a marginal effect of medication load in theta oscillatory activity, but importantly, FRN was not affected by medication. This dissociation between the differential effect of medication in theta (marginal) and FRN (no effect) might be explained by the poorer temporal resolution of theta time-frequency analysis, which might include not just FRN, but also other components such as P300. In addition it is not possible to compare this effect with previous study on BPD and FRN/ERN (de Bruijn et al., 2007, Schuermann et al., 2011) because they did not study oscillatory activity. Nevertheless, in the present study differences in FRN between controls and BDP are not affected by medication evidencing a dysfunctional reward processing in BPD patients, concretely in the negative feedback processing which might lead to deficits in learning and decision making due to an impaired capacity to elicit correct expectations and predictions. These results contribute to understanding the BPD psychopathology supporting emotional instability as one of the core features of the disorder. Furthermore in a clinical setting, where a common cost-benefits analysis is required, our results could contribute to a better approach to several important aspects such as the building of therapeutic alliance process (e.g. integrating it in the validation work), drug compliance and self-regulation training. 
NEGATIVE REWARD EXPECTATIONS IN BPD

\section{Acknowledgments}

This research was funded by the "Fundació La Marató de TV3" (2009-092410), Spanish Ministry of Science to JMP (PSI2009-09101 and PSI2012-37472) and ARF (PSI200803901). JMP is supported by the Ramon y Cajal Grant (RYC-2007-01614). 


\section{References}

Abler, B., Grön, G., Hartmann, A., Metzger, C., \& Walter, M. (2012). Modulation of frontostriatal interaction aligns with reduced primary reward processing under serotonergic drugs. The Journal of neuroscience, 32(4), 1329-1335. doi:10.1523/JNEUROSCI.5826-11.2012

Agrawal, H. R., Gunderson, J., Holmes, B. M., \& Lyons-Ruth, K. (2004). Attachment studies with borderline patients: a review. Harvard Review of Psychiatry, 12(2), 94-104. doi:10.1080/10673220490447218

Almeida, J. R. C., Akkal, D., Hassel, S., Travis, M. J., Banihashemi, L., Kerr, N., Kupfer, D., Phillips, M. L. (2009).Reduced gray matter volume in ventral prefrontal cortex but not amygdala in bipolar disorder: significant effects of gender and trait anxiety. Psychiatry research, 171(1), 54-68. doi:10.1016/j.pscychresns.2008.02.001

Amodio, D. M., Master, S. L., Yee, C. M., \& Taylor, S. E. (2008). Neurocognitive components of the behavioral inhibition and activation systems: implications for theories of self-regulation. Psychophysiology, 45(1), 11-19. doi:10.1111/j.14698986.2007.00609.x

APA. (2000). Diagnostic and Statistical Manual of Mental Disorders, Fourth Edition: $D S M-I V-T R \circledast$. American Psychiatric Pub.

Bandelow, B., Schmahl, C., Falkai, P., \&Wedekind, D. (2010). Borderline personality disorder: a dysregulation of the endogenous opioid system? Psychological Review, 117(2), 623-636. doi:10.1037/a0018095

Barrachina, J., Soler, J., Campins, M. J., Tejero, A., Pascual, J. C., Alvarez, E., ... Pérez Sola, V. (2004). Validation of a Spanish version of the Diagnostic Interview for Bordelines-Revised (DIB-R). Actas Españolas De Psiquiatría, 32(5), 293-298. 
Camara, E., Krämer, U. M., Cunillera, T., Marco-Pallarés, J., Cucurell, D., Nager, W., ...Münte, T. F. (2010). The effects of COMT (Val108/158Met) and DRD4 (SNP -521) dopamine genotypes on brain activations related to valence and magnitude of rewards. Cerebral Cortex (New York, N.Y.: 1991), 20(8), 1985-1996. doi:10.1093/cercor/bhp263

Camara, E., Rodriguez-Fornells, A., Ye, Z., \&Münte, T. F. (2009). Reward networks in the brain as captured by connectivity measures. Frontiers in Neuroscience, 3(3), 350-362. doi:10.3389/neuro.01.034.2009

Caravaglios G, Natalè E, Ferraro G, Fierro B, Raspanti G, Daniele O. (2001). Auditory event-related potentials (P300) in epileptic patients. Neurophysiologie Clinique/Clinical Neurophysiology, 31(2):121-129. doi:10.1016/S09877053(01)00252-0

Carpenter, R. W., \&Trull, T. J. (2013). Components of emotion dysregulation in borderline personality disorder: a review. Current psychiatry reports, 15(1), 335.doi:10.1007/s11920-012-0335-2

Cavanagh, J. F., Cohen, M. X., \& Allen, J. J. B. (2009). Prelude to and resolution of an error: EEG phase synchrony reveals cognitive control dynamics during action monitoring. The Journal of Neuroscience: The Official Journal of the Society for Neuroscience, 29(1), 98-105. doi:10.1523/JNEUROSCI.4137-08.2009

Chase, H. W., Swainson, R., Durham, L., Benham, L., \& Cools, R. (2011). Feedbackrelated negativity codes prediction error but not behavioral adjustment during probabilistic reversal learning. Journal of Cognitive Neuroscience, 23(4), 936946. doi:10.1162/jocn.2010.21456 
Clark, D. A., \& Beck, A. T. (2010). Cognitive theory and therapy of anxiety and depression: convergence with neurobiological findings. Trends in Cognitive Sciences, 14(9), 418-424. doi:10.1016/j.tics.2010.06.007

Cohen, M. X., Elger, C. E., \& Ranganath, C. (2007). Reward expectation modulates feedback-related negativity and EEG spectra. NeuroImage, 35(2), 968-978. doi:10.1016/j.neuroimage.2006.11.056

Corr. P.J. (2002). J. A. Gray's reinforcement sensitivity theory and frustrative non reward: a theoretical note on expectancies in reactions to rewarding stimuli. Personality and Individual Differences, 32(7), 1247-1253. doi:10.1016/S01918869(01)00115-5

Corr, P. J. (2004). Reinforcement sensitivity theory and personality. Neuroscience and Biobehavioral Reviews, 28(3), 317-332. doi:10.1016/j.neubiorev.2004.01.005

Crowell, S. E., Beauchaine, T. P., \& Linehan, M. M. (2009). A biosocial developmental model of borderline personality: Elaborating and extending Linehan's theory. Psychological Bulletin, 135(3), 495-510. doi:10.1037/a0015616

Davis, J. M., \& Chen, N. (2004). Dose response and dose equivalence of antipsychotics. Journal of clinical psychopharmacology, 24(2), 192-208.

de Bruijn, E. R. A., Grootens, K. P., Verkes, R. J., Buchholz, V., Hummelen, J. W., \& Hulstijn, W. (2006). Neural correlates of impulsive responding in borderline personality disorder: ERP evidence for reduced action monitoring. Journal of Psychiatric Research, 40(5), 428-437. doi:10.1016/j.jpsychires.2005.09.004

Dougherty, D. M., Bjork, J. M., Huckabee, H. C., Moeller, F. G., \& Swann, A. C. (1999). Laboratory measures of aggression and impulsivity in women with borderline personality disorder. Psychiatry Research, 85(3), 315-326. 
Falkenstein, M., Hohnsbein, J., Hoormann, J., \&Blanke, L. (1991). Effects of crossmodal divided attention on late ERP components. II. Error processing in choice reaction tasks. Electroencephalography and Clinical Neurophysiology, 78(6), 447-455. doi:10.1016/0013-4694(91)90062-9

First, M. B., \& Gibbon, M. (1997).User's Guide for the Structured Clinical Interview for DSM-IV Axis I Disorders SCID-I: Clinician Version. American Psychiatric Pub.

Folstein, J.R., \& Van Pettern, C. (2008). Influence of cognitive control and mismatch on the N2 component of the ERP: A review. Psychophysiology, 45(2), 152-170. doi:10.1111/j.1469-8986.2007.00602.x

Fonagy, P., \& Bateman, A. W. (2006).Mechanisms of change in mentalization-based treatment of BPD. Journal of Clinical Psychology, 62(4), 411-430. doi:10.1002/jclp.20241

Friedel, R. O. (2004). Dopamine dysfunction in borderline personality disorder: a hypothesis. Neuropsychopharmacology: Official Publication of the American College of Neuropsychopharmacology, 29(6), 1029-1039. doi:10.1038/sj.npp.1300424

Gehring, W. J., Goss, B., Coles, M. G. H., Meyer, D. E., \& Donchin, E. (1993).A Neural System for Error Detection and Compensation.Psychological Science, 4(6), 385-390. doi:10.1111/j.1467-9280.1993.tb00586.x

Gehring, W. J., \& Willoughby, A. R. (2002).The medial frontal cortex and the rapid processing of monetary gains and losses. Science (New York, N.Y.), 295(5563), 2279-2282. doi:10.1126/science.1066893 
Giorgetta C., Grecucci, A., Zuanon, S., Perini, L., Balestrieri, M., Bonini, N., Sanfey, A.G., \& Brambilla, P. (2012). Reduced risk-taking behavior as a trait feature of anxiety. Emotion. 12(6),1373-1383.

Glenn, C. R., \& Klonsky, E. D. (2009). Emotion dysregulation as a core feature of borderline personality disorder. Journal of Personality Disorders, 23(1), 20-28. doi:10.1521/pedi.2009.23.1.20

Haaland, V. Ø., \& Landrø, N. I. (2007). Decision making as measured with the Iowa Gambling Task in patients with borderline personality disorder. Journal of the International Neuropsychological Society: JINS, 13(4), 699-703. doi:10.1017/S1355617707070890

Hajcak, G., Moser, J. S., Holroyd, C. B., \& Simons, R. F. (2007). It's worse than you thought: The feedback negativity and violations of reward prediction in gambling tasks. Psychophysiology, 44(6), 905-912. doi:10.1111/j.14698986.2007.00567.x

Hajihosseini, A., \& Holroyd, C.B. (2013). Frontal midline theta and N200 amplitude reflect complementary information about expectancy and outcome evaluation. Psychophysiology. 250(6), 550-562. doi: 10.1111/psyp.12040.

Hamilton, M. (1960).A Rating Scale for Depression. Journal of Neurology, Neurosurgery, and Psychiatry, 23(1), 56-62.

Holroyd, C. B., \& Coles, M. G. H. (2002). The neural basis of human error processing: reinforcement learning, dopamine, and the error-related negativity. Psychological Review, 109(4), 679-709.

Holroyd, C.B. (2004). A note on the oddball N200 and feedback ERN. In M. Ullsberger \& M. Falkenstein (Eds.), Errors, conflicts, and the brain: Current opinions on response monitoring. Leipzig: MPI of Cognitive Neuroscience. 
Holroyd, C. B., Pakzad-Vaezi, K.L., \& Krigolson, O.E. (2008). The feedback correctrelated positivity: Sensitivity of the event-related brain potential to unexpected positive feedback. Psychophysiology, 45(5), 688-697. doi: 10.1111/j.14698986.2008.00668.x

Insel, T. R., \& Young, L. J. (2001).The neurobiology of attachment. Nature Reviews. Neuroscience, 2(2), 129-136. doi:10.1038/35053579

Johannes, S., Wieringa, B. M., Nager, W., Dengler, R., \& Münte, T.F. (2001). Oxazepam alters action monitoring. Psychopharmacology, 155(1), 100-106

Kirkpatrick, T., Joyce, E., Milton, J., Duggan, C., Tyrer, P., \& Rogers, R. D. (2007).Altered Emotional Decision-Making in Prisoners with Borderline Personality Disorder. Journal of Personality Disorders, 21(3), 243-261. doi:10.1521/pedi.2007.21.3.243

Lakatos, K., Toth, I., Nemoda, Z., Ney, K., Sasvari-Szekely, M., \&Gervai, J. (2000). Dopamine D4 receptor (DRD4) gene polymorphism is associated with attachment disorganization in infants. Molecular Psychiatry, 5(6), 633-637.

Lawrence, K. A., Allen, J. S., \& Chanen, A. M. (2010). Impulsivity in borderline personality disorder: reward-based decision-making and its relationship to emotional distress. Journal of Personality Disorders, 24(6), 786-799. doi:10.1521/pedi.2010.24.6.785

Lieb, K., Zanarini, M. C., Schmahl, C., Linehan, M. M., \& Bohus, M. (2004). Borderline personality disorder. The Lancet, 364(9432), 453-461. doi:10.1016/S0140-6736(04)16770-6

Linehan, M. (1993). Cognitive-Behavioral Treatment of Borderline Personality Disorder. Guilford Press. 
Marco-Pallares, J., Cucurell, D., Cunillera, T., García, R., Andrés-Pueyo, A., Münte, T. F., \& Rodríguez-Fornells, A. (2008).Human oscillatory activity associated to reward processing in a gambling task. Neuropsychologia, 46(1), 241-248. doi:10.1016/j.neuropsychologia.2007.07.016

Marco-Pallarés, J., Cucurell, D., Cunillera, T., Krämer, U. M., Càmara, E., Nager, W., ...Rodriguez-Fornells, A. (2009). Genetic variability in the dopamine system (dopamine receptor D4, catechol-O-methyltransferase) modulates neurophysiological responses to gains and losses. Biological Psychiatry, 66(2), 154-161. doi:10.1016/j.biopsych.2009.01.006

Masaki, H., Takeuchi, S., Gehring, W. J., Takasawa, N., \& Yamazaki, K. (2006). Affective-motivational influences on feedback-related ERPs in a gambling task. Brain Research, 1105(1), 110-121. doi:10.1016/j.brainres.2006.01.022

Mauchnik, J., \&Schmahl, C. (2010).The latest neuroimaging findings in borderline personality disorder. Current Psychiatry Reports, 12(1), 46-55. doi:10.1007/s11920-009-0089-7

Miltner, W. H. R., Braun, C. H., \& Coles, M. G. H. (1997). Event-related brain potentials following incorrect feedback in a time-estimation task: Evidence for a \&\#8220;generic\&\#8221; neural system for error detection. Journal of Cognitive Neuroscience, 9(6), 788-798. doi:10.1162/jocn.1997.9.6.788

Minzenberg, M. J., Poole, J. H., \&Vinogradov, S. (2008). A neurocognitive model of borderline personality disorder: effects of childhood sexual abuse and relationship to adult social attachment disturbance. Development and Psychopathology, 20(1), 341-368. doi:10.1017/S0954579408000163 
Mitchell, D. G. V. (2011). The nexus between decision making and emotion regulation: A review of convergent neurocognitive substrates. Behavioural Brain Research, 217, 215-231.

Pérez-Prieto, F., Alvarez, I., Monros, P., Sarria, C., Pérez-Marín, E., \& et al. (2008). Adaptación española de la SCID-II. Valencia.

Pedrao, G., Mallorquí, A., Cucurell, D., Marco-Pallarés, J. \& Rodríguez-Fornells, A. (2013). Neurophysiological differences in reward processing in anhedonics. Cognitive Affective and Behavioral Neuroscience, 13(1), 102-115.

Prossin, A. R., Love, T. M., Koeppe, R. A., Zubieta, J.-K., \& Silk, K. R. (2010).Dysregulation of regional endogenous opioid function in borderline personality disorder. The American Journal of Psychiatry, 167(8), 925-933. doi:10.1176/appi.ajp.2010.09091348

Riba, J., Rodriguez-Fornells, A., Munte, T.F., \& Barbanoj, M.J. (2005). A neurophysiological study of the detrimental effects of alprazolam on human action monitoring. Cognitive Brain Research, 25, 554-565.

Ridderinkhof, K. R., Ullsperger, M., Crone, E. A., \& Nieuwenhuis, S. (2004). The role of the medial frontal cortex in cognitive control. Science (New York, N.Y.), 306(5695), 443-447. doi:10.1126/science.1100301

Ruchsow, M., Walter, H., Buchheim, A., Martius, P., Spitzer, M., Kächele, H., ... Kiefer, M. (2006). Electrophysiological correlates of error processing in borderline personality disorder. Biological Psychology, 72(2), 133-140. doi:10.1016/j.biopsycho.2005.08.006

Sackeim, H. A. (2001). The definition and meaning of treatment-resistant depression. The Journal of clinical psychiatry, 62 (Suppl 16), 10-17 
Santesso, D. L., Dzyundzyak, A., \& Segalowitz, S. J. (2011). Age, sex and individual differences in punishment sensitivity: factors influencing the feedback-related negativity. Psychophysiology, 48(11), 1481-1489. doi:10.1111/j.14698986.2011.01229.x

Schuermann, B., Kathmann, N., Stiglmayr, C., Renneberg, B., \& Endrass, T. (2011). Impaired decision making and feedback evaluation in borderline personality disorder. Psychological $\quad$ Medicine, $\quad 41(9), \quad$ 1917-1927. doi:10.1017/S003329171000262X

Schultz, W. (1998).Predictive reward signal of dopamine neurons. Journal of Neurophysiology, 80(1), 1-27.

Steele, H., \&Siever, L. (2010). An attachment perspective on borderline personality disorder: advances in gene-environment considerations. Current Psychiatry Reports, 12(1), 61-67. doi:10.1007/s11920-009-0091-0

Strathearn, L., \& Mayes, L. C. (2010). Cocaine addiction in mothers: potential effects on maternal care and infant development. Annals of the New York Academy of Sciences, 1187, 172-183. doi:10.1111/j.1749-6632.2009.05142.x

Strauss, G. P., Frank, M. J., Waltz, J. A., Kazanova, Z., Herbener, E. S. \& Gold, J. M. (2011). Deficits in positive reinforcement learning and uncertainty-driven exploration are associated with distinct aspects of negative symptoms in schizophrenia. Biological Psychiatry, 69, 424-431. doi: 10.1016/j.biopsych.2010.10.015

Talmi, D., Fuentemilla, L., Litvak, V., Duzel, E., \& Dolan, R. J. (2012). An MEG signature corresponding to an axiomatic model of reward prediction error. NeuroImage, 59(1), 635-645. doi:10.1016/j.neuroimage.2011.06.051 
Torrubia, R., Ávila, C., Moltó, J., \& Caseras, X. (2001). The Sensitivity to Punishment and Sensitivity to Reward Questionnaire (SPSRQ) as a measure of Gray's anxiety and impulsivity dimensions. Personality and Individual Differences, 31(6), 837-862. doi:10.1016/S0191-8869(00)00183-5

Unger, K., Heinz, S., \& Kray, J., (2012) Punishment sensitivity modulates the processing of negative feedback but not error-induced learning. Frontiers in Human Neuroscience, 6, 186.

Vederman, A. C., Weisenbach, S. L., Rapport, L. J., Leon, H. M., Haase, B. D., Franti, L. M., ... McInnis, M. G. (2012). Modality-specific alterations in the perception of emotional stimuli in Bipolar Disorder compared to Healthy Controls and Major Depressive Disorder. Cortex, 48(8), 1027-1034. doi:10.1016/j.cortex.2011.03.017

Völlm, B., Richardson, P., McKie, S., Elliott, R., Dolan, M., \&Deakin, B. (2007). Neuronal correlates of reward and loss in Cluster B personality disorders: a functional magnetic resonance imaging study. Psychiatry Research, 156(2), 151-167. doi:10.1016/j.pscychresns.2007.04.008

Vrticka, P., Andersson, F., Grandjean, D., Sander, D., \&Vuilleumier, P. (2008). Individual attachment style modulates human amygdala and striatum activation during social appraisal. PloS One, 3(8), e2868. doi:10.1371/journal.pone.0002868

Zanarini, M. C., Frankenburg, F. R., Reich, D. B., \& Fitzmaurice, G. (2010). The 10year course of psychosocial functioning among patients with borderline personality disorder and axis II comparison subjects. Acta Psychiatrica Scandinavica, 122(2), 103-109. doi:10.1111/j.1600-0447.2010.01543 

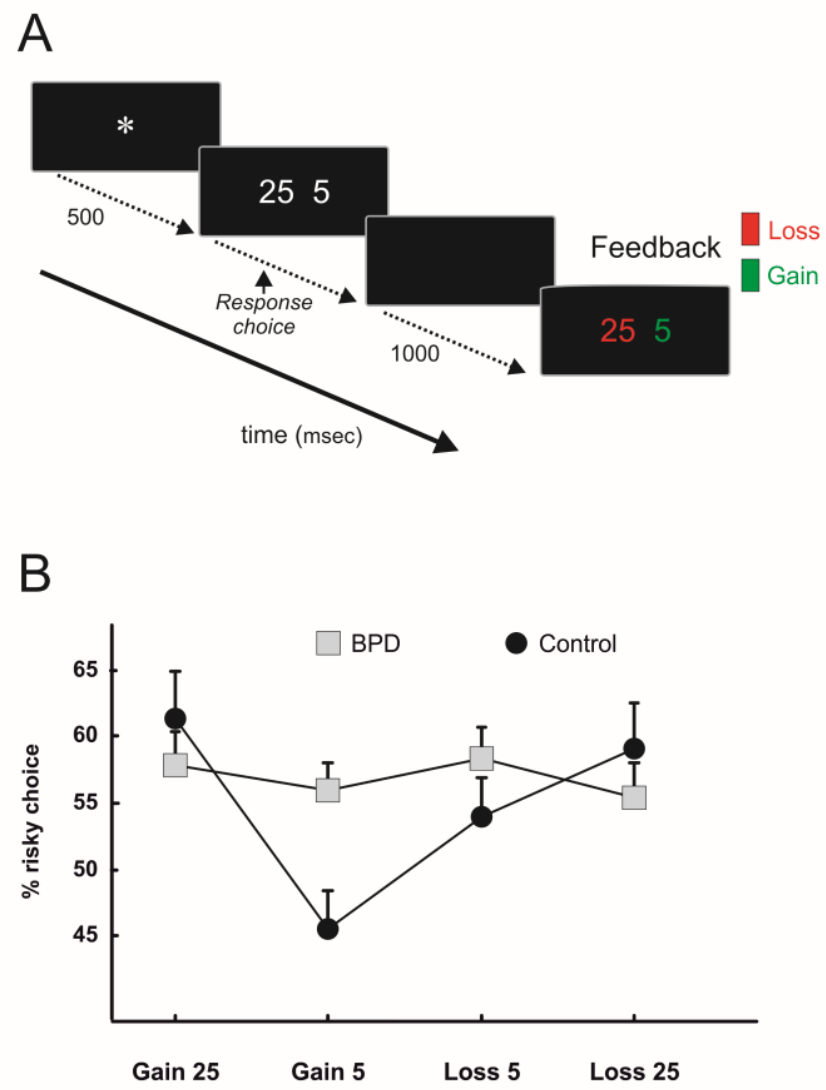

Figure 1. A. Gambling paradigm used in the experiment. B. Effect of previous trial (n$1, x$ axis) in the risk pattern observed in the following trial (percent of choice of 25 instead of 5), in the BPD and control groups. The lines represent the percent of behavioral risky choices (total bets to 25) in function of the feedback received in the previous trial (four possible outcomes: gain 25, gain 5, loss 5 and loss 25). Notice the lack of sequential adjustment of risk patterns in the BPD patients when compared to the control group. 
A Control
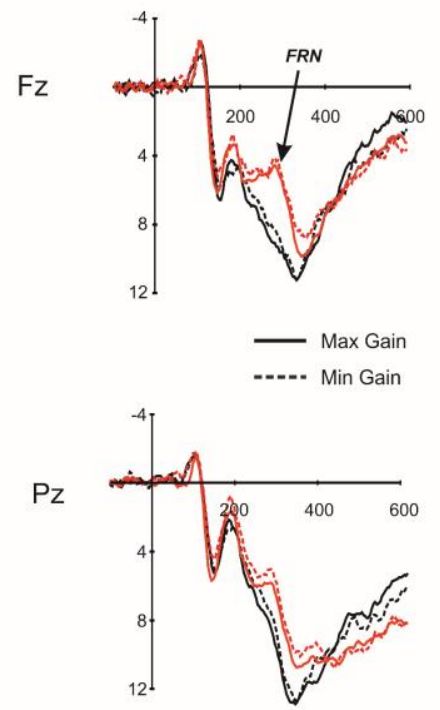

BPD

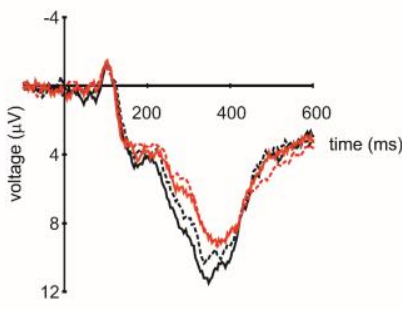

— Max Loss

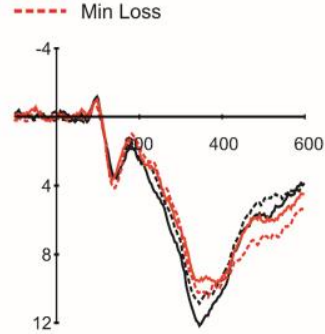

B
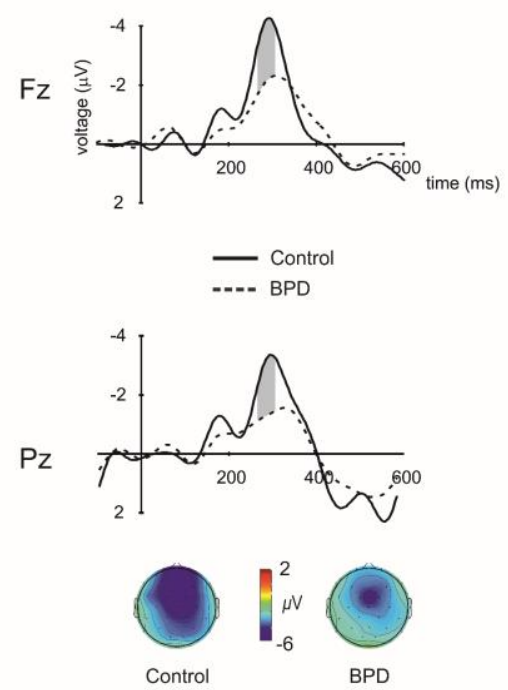

Figure 2. A. Event-Related Potentials associated to the four studied types of feedback: maximum gain (solid black), minimum gain (dashed black), maximum loss (solid red) and minimum loss (dashed red) for the control (left) and BPD group (right) at three midline electrode locations (Fz, Cz, Pz). Notice the increase of negativity between 260 and $300 \mathrm{~ms}$ for negative feedbacks compared to positive ones in the control group (FRN). This effect is reduced in the BPD group. B. Loss minus gain difference waveform at the $\mathrm{Cz}$ electrode for the control (blue) and BPD (orange) group. For illustration purposes, activity has been filtered with a $12 \mathrm{~Hz}$ lowpass filter. Region in green indicates significant differences between groups (260-300 ms). Bottom, scalp topographical maps for the difference waveform (loss minus gain) in the green region for control (left) and BPD (right) groups. 
A

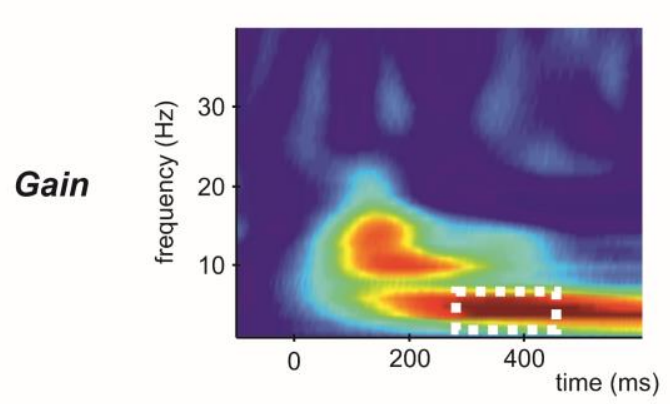

$\left(\frac{10}{2}\right.$

Loss
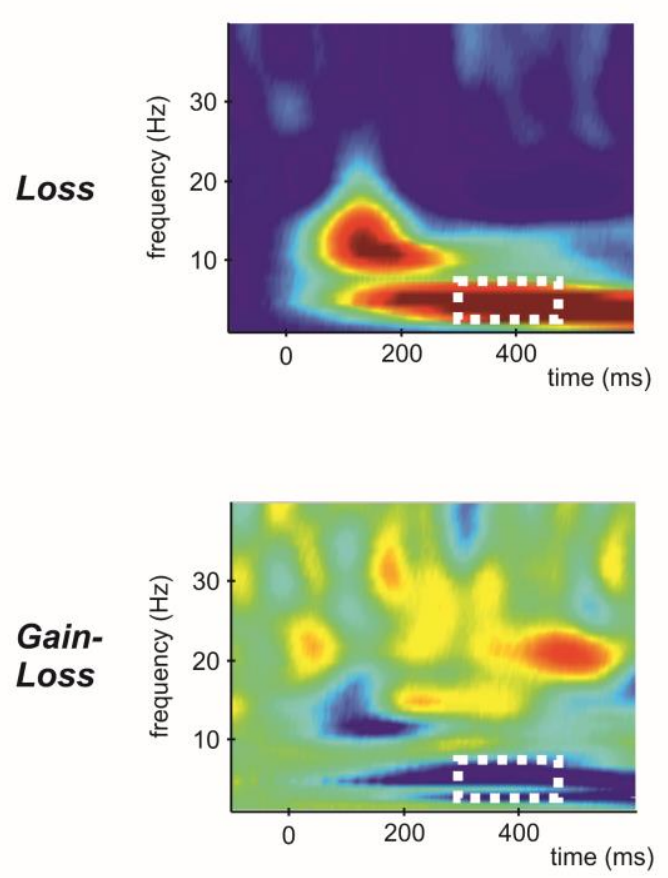

B

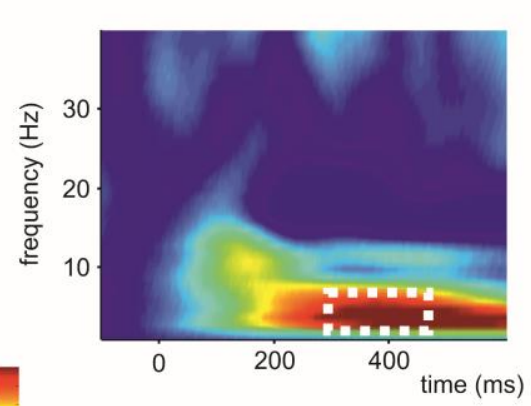

$0 \%$
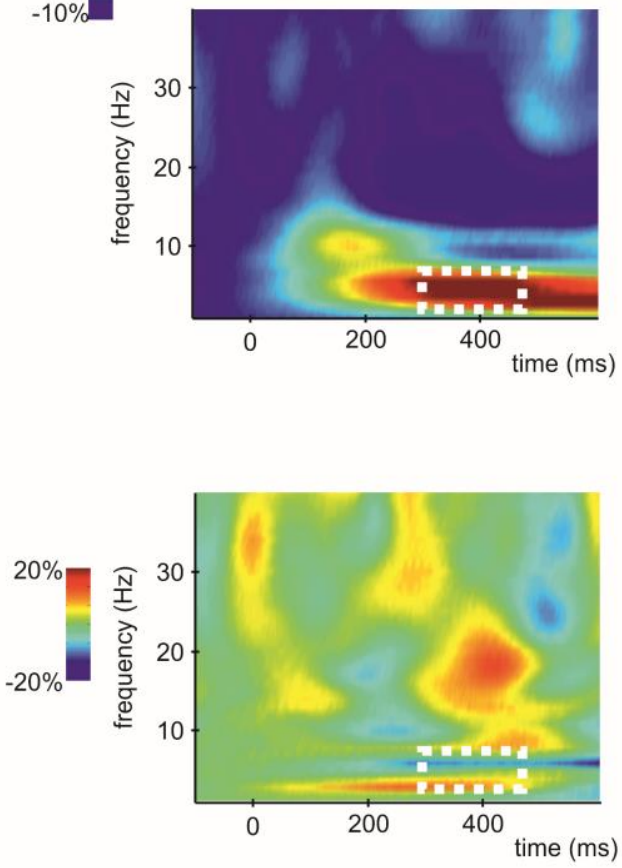

Figure 3. Time-frequency plots at the Fz electrode for (A) the control and (B) the BPD group. From top to bottom can be seen the power changes at the frequencies between 1 and $40 \mathrm{~Hz}$ of: gain, loss and gain minus loss. The white rectangle indicates the timefrequency studied area for the theta band $(3-7 \mathrm{~Hz}, 300-450 \mathrm{~ms})$. 


\section{Tables}

Table 1. Demographic characteristics, clinical and psychometric variables.

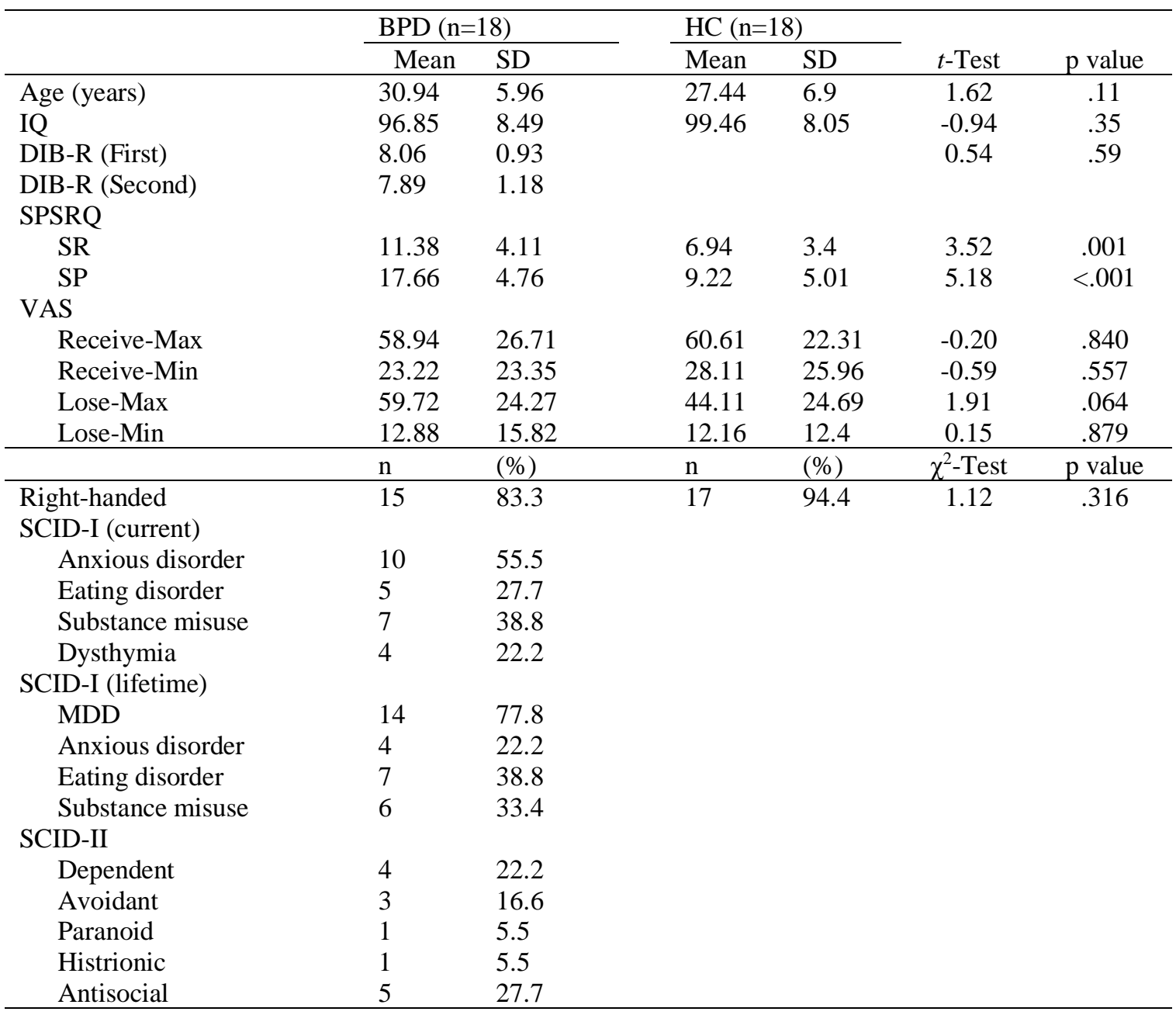

IQ, intelligence quotient, estimated through matrix reasoning, vocabulary and digits span subtests (WAIS-III); GAF, Global Assessment of Functioning; SPSRQ, Sensitivity to Punishment and Sensitivity to Reward Questionnaire; SP, Sensitivity to Punishment; SR, Sensitivity to Reward; VAS, Visual Analog Scale; DIB-R, Diagnostic Interview for Borderlines-Revised; MDD, Major Depressive Disorder. 